\title{
Picosecond dynamics of free and bound excitons in doped diamond
}

\author{
J. Barjon,,${ }^{1, *}$ P. Valvin, ${ }^{2,3}$ C. Brimont,${ }^{2,3}$ P. Lefebvre, ${ }^{2,3}$ O. Brinza, ${ }^{4}$ A. Tallaire,${ }^{4}$ J. Achard,${ }^{3}$ \\ F. Jomard, ${ }^{1}$ and M. A. Pinault-Thaury ${ }^{1}$ \\ ${ }^{1}$ Groupe d'Etude de la Matière Condensée (GEMaC), Université Versailles St. Quentin en Yvelines, CNRS, Université Paris Saclay, \\ 45 avenue des Etats-Unis, 78035 Versailles, France \\ ${ }^{2}$ Laboratoire Charles Coulomb (L2C), CNRS, UMR5221, 34095 Montpellier, France \\ ${ }^{3}$ Laboratoire Charles Coulomb (L2C), Université Montpellier 2, UMR5221, 34095 Montpellier, France \\ ${ }^{4}$ Laboratoire des Sciences des Procédés et des Matériaux (LSPM), Université Paris 13, Sorbonne Paris Cité, \\ CNRS, 93430 Villetaneuse, France
}

(Received 4 August 2014; revised manuscript received 30 December 2015; published 11 March 2016)

\begin{abstract}
The dynamics of the free-exciton capture by boron acceptors and phosphorus donors in diamond is observed in the picosecond range by time-resolved photoluminescence experiments at low temperature. The formation of boron-bound excitons is observed with a delay of $410 \mathrm{ps}$ after the formation of free excitons. For phosphorus, this delay is $120 \mathrm{ps}$. This is the result of the free-exciton capture by $\mathrm{B}^{0}$ and $\mathrm{P}^{0}$ impurities. The lifetimes of boronand phosphorus-bound excitons are measured and found to be equal to 270 and $70 \mathrm{ps}$, respectively. These values are about four orders of magnitude shorter than for the same impurities in silicon. $E_{i}$ being the ionization energy of dopants, these results scale well with the $E_{i}^{4}$ dependence of the Auger recombination rate expected for bound excitons in indirect band-gap semiconductors.
\end{abstract}

DOI: 10.1103/PhysRevB.93.115202

\section{INTRODUCTION}

Well known as a hard and shining crystal, diamond is also a semiconductor material with a unique set of physical properties [1]. With a wide band-gap energy (5.47 eV indirect), intrinsic diamond remains a good electrical insulator up to extremely high temperatures $\left(10^{10}\right.$ free carriers $/ \mathrm{cm}^{3}$ at $\left.1000^{\circ} \mathrm{C}\right)$. It can withstand extremely high electric fields before breakdown $(>10 \mathrm{MV} / \mathrm{cm})$. Its exceptional thermal conductivity (five times that of copper) is a substantial asset when it comes to the thermal management of electronic devices. Moreover, charges are extremely mobile in pure diamond (mobility $>3000 \mathrm{~cm}^{2} / \mathrm{V} \mathrm{s}$ for electrons and holes). Because of these exceptional properties, diamond is considered to be the ultimate semiconductor for electronics, and for power applications in particular. However, its practical use has been limited for a long time by the difficulties of its artificial synthesis and doping. With recent progress in chemical vapor deposition (CVD), high-purity crystals are now available $\left(<10^{14}\right.$ impurities $\left./ \mathrm{cm}^{3}\right)$. Concomitant progress in the control of both $p$ - and $n$-type doping has led to the first prototypes of $p$ - $n$ diodes [2] and bipolar transistors [3].

However, the use of diamond in electronics raises technological as well as physical issues compared to the more mature area of silicon microelectronics. Traditional electronic devices are basically built on the properties of free charge carriers, namely independent electrons and holes, without considering exciton formation, diffusion, and recombination processes. For silicon, this assumption is justified since the exciton population is most often negligible in a room-temperature operating device. This is a consequence of the low binding energy of excitons in silicon (14 meV). In diamond, the exciton binding energy is much higher $(80 \mathrm{meV})$ [4], so that

\footnotetext{
*Corresponding author: julien.barjon@uvsq.fr
}

the exciton population should be considered. Excitons may be dominant over free carrier densities in diamond devices operating at room temperature under high injection conditions, e.g., in electroluminescent or power devices. The dynamic balance between free charge and exciton populations is indeed displaced in favor of excitons under high injection conditions $[5,6]$. Then the excitonic recombination properties-which only manifest close to liquid helium temperature in silicon components $[7,8]$ - will have to be integrated in simulators for a reliable design of optoelectronic devices, and might lead to unique exciton-based operating schemes.

More fundamentally, excitons in diamond form an interesting system to experimentally investigate many-body physics. With femtosecond optics, significant advances in the understanding of free-exciton dynamics have been provided recently with ultrapure CVD diamonds studied under high injection conditions at low temperature. In particular, the interplay of free excitons with the condensation of electronhole droplets (EHDs) [9] and the formation of polyexcitons [10] have been evidenced. However, it is indisputable that doping impurities necessarily play a role in the recombination dynamics of excitons in diamond. Yet, a basic knowledge of the corresponding time constants is still missing.

In this paper, the dynamics of the free-exciton capture by boron acceptors and phosphorus donors is investigated by time-resolved photoluminescence (TRPL) spectroscopy at low temperature. Weak excitation conditions are used in order to avoid saturation effects. Bound-exciton lifetimes in the range of a few tens of picoseconds are measured, consistent with recombinations governed by Auger processes.

\section{EXPERIMENT}

The $p$ - and $n$-type diamond samples investigated in this work are homoepitaxial layers grown by chemical vapor deposition (CVD) assisted by a microwave plasma on 
(100)-oriented, high-pressure/high-temperature Ib diamond substrates. The two $p$-type epilayers are doped either with $2.5 \times 10^{16}$ boron atoms $/ \mathrm{cm}^{3}$ on a $13 \mu \mathrm{m}$ thickness-referred to here as weakly doped-or with $1.5 \times 10^{17}$ boron atoms $/ \mathrm{cm}^{3}$ on a $9.3 \mu \mathrm{m}$ thickness-referred here as highly doped. Growth conditions, secondary ion mass spectrometry (SIMS), and excellent electrical properties of the $p$-type samples are described in Ref. [11] using the labels " $A$ " and "B" for the weakly and highly doped samples, respectively. The $n$-type layer is doped with $5.1 \times 10^{16}$ phosphorus atoms $/ \mathrm{cm}^{3}$ along a thickness of $6 \mu \mathrm{m}$, also measured by SIMS. References $[12,13]$ introduce, with more details, the physical properties of the phosphorus-doped diamond.

The luminescence spectra were first recorded with high spectral resolution $(0.02 \mathrm{~nm})$ under continuous-wave (cw) electron-beam excitation, the samples being mounted on a helium cryostat at $5 \mathrm{~K}$. The cathodoluminescence (CL) setup is described in Ref. [14]. The formation and decay dynamics of excitons were further investigated by using timeresolved photoluminescence (TRPL). The diamond samples were excited at a wavelength of $200 \mathrm{~nm}$ in pulsed mode, with the fourth harmonic of a titanium-doped sapphire laser focused on a $\sim 50 \mu \mathrm{m}$ diameter spot. Optical manipulation of ultrashort laser pulses at such a small wavelength is far from being straightforward, given the highly dispersive and often absorbent materials used for lenses and windows, in addition to parasitic fluorescence. State-of-the-art optical elements and specific dielectric coatings were therefore necessary to preserve the intensity of the excitation laser and to avoid spurious detected signals. The samples were cooled in an Oxford Microstat-HiRes 2 cryostat, down to a temperature of $5 \mathrm{~K}$. The laser pulses have a $150 \mathrm{fs}$ duration and an $82 \mathrm{MHz}$ repetition rate, for an average power of $150 \mu \mathrm{W}$ measured on the weakly doped samples. This gives an energy density per pulse of about $0.1 \mu \mathrm{J} / \mathrm{cm}^{2}$.

Such conditions of extremely weak excitation are required to perform a correct analysis of bound-exciton decay times. They prevent heating effects, saturation of the bound-exciton population, and electron-hole droplet condensation. Such phenomena were observed with excitation densities above $500 \mu \mathrm{J} / \mathrm{cm}^{2}$ [15]. A $0.1 \mu \mathrm{J} / \mathrm{cm}^{2}$ excitation density being 5000 times lower, the emitted photon flux is extremely weak and requires a high-sensitivity detection system. The spectral analysis was performed with an iHR550 Horiba Jobin Yvon monochromator equipped with a 1200 grooves $/ \mathrm{mm}$ grating. On the first exit port was mounted a silicon back-illuminated CCD camera for optical alignments in quasicontinuous mode acquisition. On the second port, a new-generation Hamamatsu C10910 streak camera optimized for the ultraviolet region, with ultimate picosecond time resolution, provides the required high sensitivity for TRPL acquisition [16]. For the TRPL experiments, the spectral resolution was lower $(0.5 \mathrm{~nm})$ than in CL so as to preserve a sufficient signal-to-noise ratio. To study boron- and phosphorus-doped diamonds, the adapted time ranges were 2000 and 600 ps full time scale, respectively. Therefore, the actual temporal resolutions (full width at half maximum of the laser pulse trace) were 52 and 18 ps for the boron- and phosphorus-doped diamonds, respectively. Given the weakness of the PL signal, acquisition times of several hours were necessary.

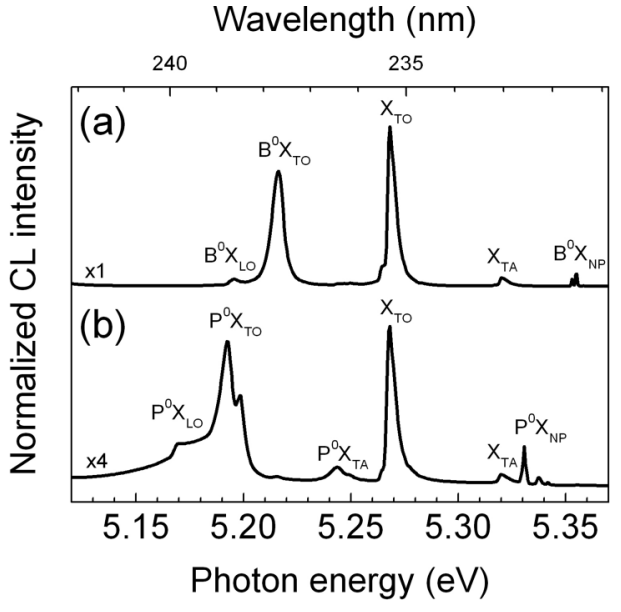

FIG. 1. High-resolution cathodoluminescence spectra under continuous excitation of diamond layers weakly doped with (a) boron and (b) phosphorus. The free exciton is labeled $X$, while neutral-boronand neutral-phosphorus-bound excitons are labeled $\mathrm{B}^{0} X$ and $P^{0} X$, respectively. Subscripts TO, TA, and LO indicate the assisting phonon involved in these indirect exciton recombinations, while NP means no phonon (NP) assistance. The sample holder temperature was $5 \mathrm{~K}$.

\section{RESULTS AND ANALYSIS}

In Fig. 1 are reported the high-resolution CL spectra under continuous electron beam excitation of the $p$ - and $n$-type samples. The free-exciton recombinations are observed with a maximum at $5.268 \mathrm{eV}$ when assisted by a transverse optical (TO) phonon. The transition is labelled $X_{\mathrm{TO}}$. Free-exciton recombinations with other assisting phonons, such as the longitudinal optical (LO) or the transverse acoustical (TA) phonons, are also observed, with much lower intensities. In the following, subscripts TO, TA, and LO indicate the assisting phonon involved in the recombination of indirect excitons.

Bound excitons are observed with maxima at 5.216 and $5.192 \mathrm{eV}$, respectively, for the neutral-boron $\left(\mathrm{B}^{0} X_{\mathrm{TO}}\right)$ and neutral-phosphorus $\left(\mathrm{P}^{0} X_{\mathrm{TO}}\right)$ bound-exciton complexes. The TO-assisted recombinations of bound excitons appear shifted at lower energy compared to the free-exciton ones. The shift corresponds to the value of the localization energy of free excitons on the neutral impurities. According to the Haynes rule [17], the deeper the dopant, the higher the localization energy of the associated bound exciton. The Haynes rule has been shown to be well verified in diamond [14]. That the electronic level of the phosphorus donor in the diamond band gap is deeper than the boron acceptor explains why $\mathrm{P}^{0} X_{\mathrm{TO}}$ recombination occurs at a lower energy than $\mathrm{B}^{0} X_{\mathrm{TO}}$.

Figure 2 presents the TRPL results. Let us first briefly describe the free-exciton formation dynamics. By extracting the time constant of the $X_{\mathrm{TO}}$ exponential growth, we found an 11 ps rise time for the free-exciton recombinations (phosphorus-doped sample; 600 ps time range). This value is close to the $7 \mathrm{ps}$ rise time of the optical setup, measured from the laser diffusion trace on the same time range. This shows that the formation of free excitons is extremely fast, within a few picoseconds.

The formation kinetics of bound excitons is much slower. For boron- and phosphorus-bound excitons, the maximum of 
(a) boron

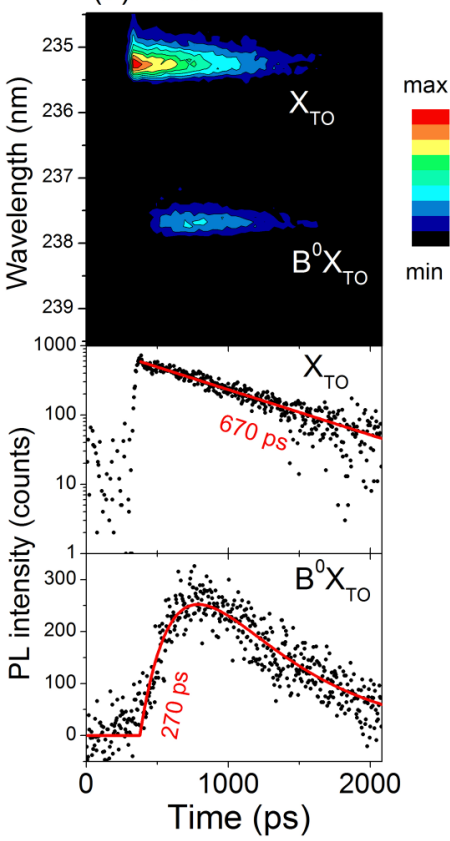

(b) phosphorus

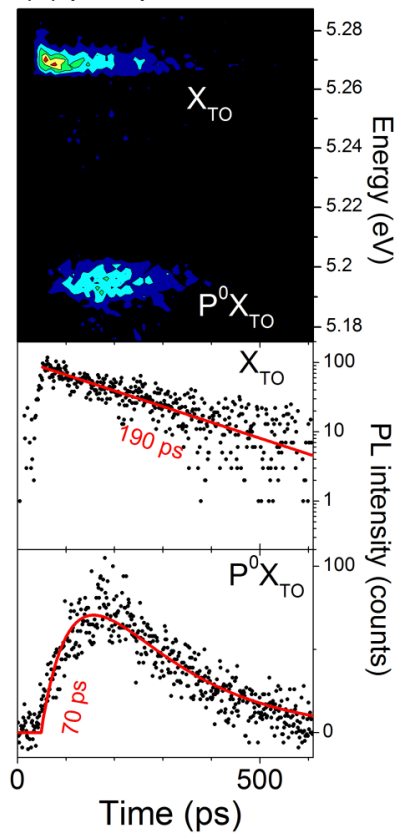

FIG. 2. Time-resolved photoluminescence (TRPL) at $5 \mathrm{~K}$ of the TO-assisted recombinations of excitons in diamond doped with (a) boron or (b) phosphorus impurities in the $10^{16}$ atoms $/ \mathrm{cm}^{3}$ range. The energy density was $\sim 0.1 \mu \mathrm{J} / \mathrm{cm}^{2}$ per pulse. The time dependence of the free exciton $\left(X_{\mathrm{TO}}\right)$ is fitted with a single exponential decay. Boundexciton recombinations $\left(\mathrm{B}^{0} X_{\mathrm{TO}}\right.$ and $\left.\mathrm{P}^{0} X_{\mathrm{TO}}\right)$ are adjusted according to Eq. (4), details being given in the text. TRPL images are plotted after an $8 \times 8$ binning.

the PL signal is reached later than the free-exciton maximum. The delays are 410 ps for boron- and 120 ps for phosphorusbound excitons-both values being much larger than the temporal resolution. Such delays are expected because the formation of bound excitons is governed by the capture of free excitons by the dopants, as explained in the following.

If a single kind of impurity is considered, the respective populations of free and bound excitons can be described with the rate equations of a three-level system: ground state, and bound- and free- exciton levels, as illustrated in Fig. 3. At low temperature, the dissociation of bound excitons is negligible $\left(k T \ll E_{\mathrm{loc}}\right)$. With the extremely low excitation conditions used for the weakly doped samples, the initial exciton density $n_{0}$ is about $2 \times 10^{14} / \mathrm{cm}^{3}$ [18], which is much lower than the impurity concentration in the samples. In these conditions, the

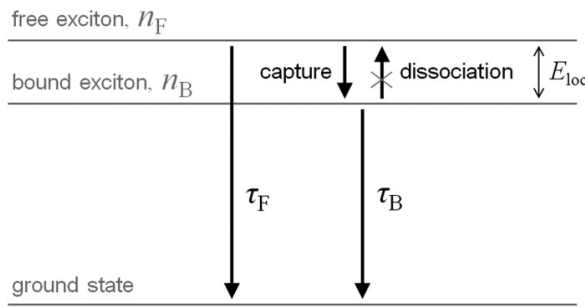

FIG. 3. Three-level scheme describing the free-exciton capture by impurities in the low-excitation regime. At low temperatures, the dissociation of bound excitons is negligible. three-level assumption is reasonable and the rate equations become particularly simple (a more general model can be found in Ref. [19]):

$$
\begin{aligned}
& \frac{d n_{\mathrm{F}}}{d t}=-\frac{n_{\mathrm{F}}}{\tau_{\mathrm{F}}}-\sigma v_{\mathrm{th}}[I] n_{\mathrm{F}}, \\
& \frac{d n_{\mathrm{B}}}{d t}=-\frac{n_{\mathrm{B}}}{\tau_{\mathrm{B}}}+\sigma v_{\mathrm{th}}[I] n_{\mathrm{F}},
\end{aligned}
$$

where $n_{\mathrm{F}}$ and $n_{\mathrm{B}}$ are the free- and bound-exciton populations, respectively. $\tau_{\mathrm{F}}$ is the free-exciton radiative lifetime and $\tau_{\mathrm{B}}$ is the bound-exciton lifetime. $\sigma$ is the capture cross section of the impurity, $v_{\text {th }}$ is the thermal velocity of free excitons, and $[I]$ is the impurity concentration in the crystal.

For free excitons (FEs), the solution of Eq. (1) gives a single exponential decay with a characteristic time constant $\tau$ such as $1 / \tau=1 / \tau_{\mathrm{F}}+\sigma v_{\mathrm{th}}[I]$. The time dependence of $X_{\text {TO }}$ in Fig. 2 is clearly consistent with this picture: It exhibits a single exponential decay with a time constant of $670 \pm 50 \mathrm{ps}$ in the boron-doped diamond sample and $190 \pm 20 \mathrm{ps}$ in the phosphorus-doped diamond sample. On the other hand, in ultrapure diamond samples, 700-800 ns free-exciton decay times were measured recently [20]. This gives the order of magnitude of $\tau_{\mathrm{F}}$, extremely long in indirect band-gap semiconductors because of the low probability of phonon-assisted radiative transitions [21]. The short FE decay times $\tau$ measured here in doped diamond indicate that $\tau$ rather corresponds to the capture time by the dopant impurities diluted in the crystal lattice:

$$
1 / \tau \approx \sigma v_{\mathrm{th}}[I]
$$

In other words, in doped diamond under weak excitation conditions, the FE decay time is governed by the dynamics of FE trapping on impurities. As a result, the free-exciton capture time is inversely proportional to the dopant concentration, as discussed later.

With a translational effective mass $0.76 m_{0}$ for excitons in diamond [22], $v_{\text {th }}=1.6 \times 10^{6} \mathrm{~cm} / \mathrm{s}$ at $5 \mathrm{~K}$. Assuming that the SIMS analysis gives the acceptor and donor concentrations in diamond (well accepted for boron and demonstrated for phosphorus in Ref. [12]), the free-exciton capture cross sections of $\mathrm{B}^{0}$ and $\mathrm{P}^{0}$ in diamond are $4 \times 10^{-14}$ and $6 \times 10^{-14} \mathrm{~cm}^{2}$, respectively. The order of magnitude is consistent with the reported values in silicon, in the range of $10^{-14} \mathrm{~cm}^{2}[23,24]$. These values, measured in diamond, are fundamental for understanding exciton dynamics in diamond-based optoelectronics and many-body physics.

For bound excitons (BEs), the temporal analysis is not as straightforward as for free excitons. Designating $\tau_{\mathrm{B}}$ the $\mathrm{BE}$ lifetime, the solutions of Eqs. (1) and (2) give the following time dependence for the BE population, $n_{\mathrm{B}}$ [25]:

$$
n_{\mathrm{B}}(t) \alpha \frac{1}{\tau_{\mathrm{B}}^{-1}-\tau^{-1}}\left[\exp \left(\frac{-t}{\tau}\right)-\exp \left(\frac{-t}{\tau_{\mathrm{B}}}\right)\right] .
$$

Depending on the values of $\tau$ and $\tau_{B}$, Eq. (4) indicates that the BE luminescence rise time is governed by the shorter lifetime and the BE luminescence decay by the longer one. In Fig. 2 for both boron and phosphorus, the BE luminescence decay exhibits a dynamics at long times which corresponds to the decay time $\tau$ measured previously for FEs. This means that 
the $\mathrm{BE}$ lifetimes are shorter and therefore correspond to the rising of the BE luminescence. Curves resulting from Eq. (4) are plotted in Fig. 2 by fixing the value of $\tau$ measured from the free-exciton decay and by fitting the values of $\tau_{\mathrm{B}}$. The extracted lifetimes $\tau_{\mathrm{B}}$ of the boron- and phosphorus-bound excitons are 270 and $70 \mathrm{ps,}$, respectively [26]. To the best of our knowledge, these are the shortest reported values for bound exciton lifetimes in indirect semiconductors.

According to Eq. (3), the free-exciton lifetime should become shorter at high doping. Then following Eq.(4), the interpretation of the bound-exciton signal may become reversed at high doping: the $\mathrm{BE}$ rise-time might correspond to the FE lifetime while the BE decay-time might correspond to the BE lifetime. Complementary experiments have been performed as a validity test.

Figure 4 reports experiments performed on the highly doped $p$-type sample. In Fig. 4(a), the $X_{\text {TO }}$ free-exciton decay time is $115 \mathrm{ps}$ for $[\mathrm{B}]=1.5 \times 10^{7}$ atoms $/ \mathrm{cm}^{3}$. This is about six times shorter than the 670 ps decay time measured for $[\mathrm{B}]=2.5 \times$ $10^{16}$ atoms $/ \mathrm{cm}^{3}$ in Fig. 2(a). At high boron concentration, the capture time of free excitons is strongly decreased and almost follows the $1 / \tau \approx \sigma v_{\mathrm{th}}[I]$ relationship.

Analyzing the temporal trace of the bound-exciton signal at high doping with Eq. (4), we found a rise time for $\mathrm{B}^{0} X_{\mathrm{TO}}$ in Fig. 4(a) corresponding to the 115 ps free-exciton decay time, measured above on $X_{\mathrm{TO}}$. This means that the $\mathrm{BE}$ rise time now corresponds to the FE lifetime and the BE decay to the BE lifetime. At high doping, the analysis of the $\mathrm{BE}$ temporal profiles is indeed reversed compared to Fig. 2.
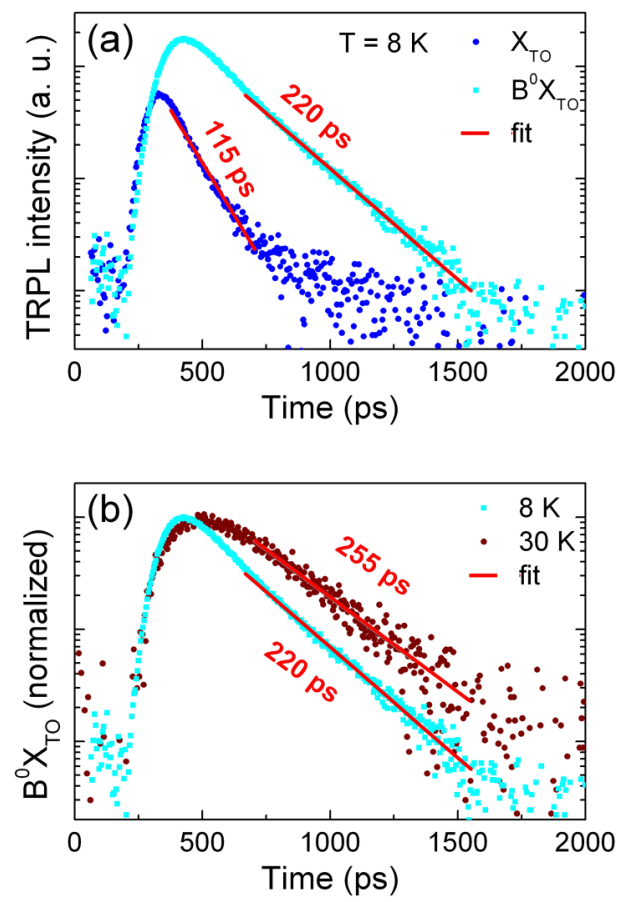

FIG. 4. (a) Temporal profiles at $8 \mathrm{~K}$ of the free-exciton $\left(X_{\mathrm{TO}}\right)$ and the neutral-boron-bound-exciton $\left(\mathrm{B}^{0} X_{\mathrm{TO}}\right)$ recombinations in a $p$ type diamond of higher boron content $[\mathrm{B}]=1.5 \times 10^{17}$ atoms $/ \mathrm{cm}^{3}$; (b) comparison of the bound-exciton decay time at 8 and $30 \mathrm{~K}$. The energy density was $\sim 0.6 \mu \mathrm{J} / \mathrm{cm}^{2}$ per laser pulse.
This high-doping situation simplifies the boron-bound-exciton lifetime determination: The $\tau_{\mathrm{B}}$ value is extracted from a single exponential fit of the BE decay time in Fig. 4(a), giving 220 \pm 50 ps. This is in good agreement with the value $270 \pm$ $50 \mathrm{ps}$ measured previously in the weakly boron-doped sample, given the experimental and fitting uncertainties. Incidentally, the bound-exciton lifetime appears almost independent of the doping level. This is coherent because the bound-exciton lifetime is expected to be a characteristic of the impurity involved in the impurity-exciton complex [19].

Insights on the role of temperature on the bound-exciton lifetime could also be gained from Fig. 4(b). At $8 \mathrm{~K}$, we found a neutral-boron-bound-exciton lifetime of $220 \pm 50 \mathrm{ps}$, while at $30 \mathrm{~K}$ we found $255 \pm 50 \mathrm{ps}$. The bound-exciton lifetime $\tau_{\mathrm{B}}$ appears to be only weakly dependent on the temperature. This preliminary result on the temperature dependence will have to be confirmed by a more systematic study.

\section{DISCUSSION}

In Table I, it is striking that the BE lifetimes are extremely short in diamond compared to silicon: They are found in the range of a few tens of picosecond, i.e., about four orders of magnitude shorter than in silicon. Short BE lifetimes were also measured with $6 \mathrm{H}-\mathrm{SiC}: \mathrm{N}$, but in the nanosecond range [27]. In indirect band-gap semiconductors, the decay of bound excitons is usually governed by nonradiative Auger recombination [28,29]. Briefly describing the phenomenon in the case of the neutral-phosphorus-bound-exciton complex, $\mathrm{P}^{0} X=\left(\mathrm{P}^{+}, e^{-}, h^{+}, e^{-}\right)$to be specific, this Auger process is written $\mathrm{P}^{0} X \rightarrow \mathrm{P}^{+}+e^{-}$. The hole recombines with one of the two electrons, and the recombination energy (comparable to the band gap) is transferred to the remaining electron of the $\mathrm{P}^{0} X$ complex. The emitted electron is then excited high in the conduction band and further relaxes nonradiatively by multiphonon emission. It is clear that the more tightly bound the exciton complex $\left(\mathrm{P}^{+}, e^{-}, h^{+}, e^{-}\right)$, the higher its Auger recombination rate. Simple models (see, for instance, Ref. [30]) predict a scale law where the Auger lifetime is inversely proportional to $E_{i}^{4}$, with $E_{i}$ the ionization energy of the dopant. In diamond, boron and phosphorus dopants are about ten times deeper than in silicon (see Table I). Therefore, the observed $\sim 10^{4}$-times-shorter BE lifetimes do scale well with the $E_{i}^{4}$ dependence of the Auger recombination rate of bound excitons. We have also observed a weak dependency of the bound-exciton lifetime on the temperature, as expected

TABLE I. Ionization energies of dopants and their corresponding bound-exciton lifetimes in silicon and diamond. Diamond lifetimes as measured in Fig. 2.

\begin{tabular}{|c|c|c|c|c|}
\hline & \multicolumn{2}{|c|}{ Silicon [29] } & \multicolumn{2}{|c|}{ Diamond (this work) } \\
\hline & B & $\mathrm{P}$ & B & $\mathrm{P}$ \\
\hline $\begin{array}{l}\text { Ionization energy } \\
E_{i}(\mathrm{meV})\end{array}$ & 44.3 & 45.5 & 380 & 600 \\
\hline $\begin{array}{l}\text { Bound-exciton lifetime } \\
\tau_{\mathrm{B}}(\mathrm{ns})\end{array}$ & 1055 & 272 & $0.27 \pm 0.05$ & $0.07 \pm 0.03$ \\
\hline
\end{tabular}


for Auger recombinations [31]. From these, we conclude that bound-exciton recombinations in diamond are governed by the nonradiative excitonic Auger recombinations. The picosecond-range lifetimes of bound excitons in diamond appear as a direct consequence of the deep character of its substitutional dopants.

Paradoxically, the deep character of dopants in wide bandgap semiconductors makes the dynamics of bound excitons quite simple. In silicon, a single exciton bound to a single impurity can trap other free excitons, leading to the formation of excitonic molecules, the so-called bound-multi-exciton complexes (BMECs) [23]. The presence of BMECs makes the capture dynamics of free excitons by doping impurities a rather complex process in silicon. In diamond, BMECs have not been observed, even at high excitation, contrary to their intrinsic analogs, the polyexcitons [10]. The extremely short Auger decay times of bound excitons evidenced in this work might be responsible for the instability of BMECs in diamond. From the characterization point of view, the consequences of BMEC recombinations are important if one intends to estimate the densities of substitutional dopants by analyzing luminescence spectra under continuous excitation [32,33]. In silicon, the established cw-PL method based on the ratio between boundand free-exciton signals requires sufficiently low laser powers to avoid any BMEC formation [34]. In diamond the same method has been shown to be much more robust upon the excitation conditions [35].

\section{CONCLUSIONS}

The capture dynamics of free excitons by dopants has been observed in $p$ - and $n$-type diamond. The capture cross sections of boron acceptors and phosphorus donors have been deduced. The lifetimes of subsequently formed bound excitons are found to be extremely short, in the range of a few tens of picoseconds. The excitonic Auger recombination process accounts well for such short bound-exciton lifetimes, which are an indirect consequence of the high ionization energies of boron and phosphorus dopants in diamond. Substitutional dopants generally produce deep electronic levels in wide bandgap semiconductors. These results are then expected to be of general scope for wide and indirect band-gap semiconductors, and should apply to other materials of this family.

\section{ACKNOWLEDGMENTS}

The authors are grateful to Christèle Vilar for her technical help on SEM-CL experiments and to Jacques Chevallier for his critical reading of the manuscript. The DIAMONIX and DIAMONIX2 French research projects, approved by the competitiveness cluster of Aerospace Valley and funded by the Unique Interministerial Fund (FUI), are acknowledged for their financial support.
[1] C. J. H. Wort and R. S. Balmer, Mater. Today 11, 22 (2008).

[2] S. Koizumi, K. Watanabe, M. Hasegawa, and H. Kanda, Science 292, 1899 (2001).

[3] H. Kato, K. Oyama, T. Makino, M. Ogura, D. Takeuchi, and S. Yamasaki, Diamond Relat. Mater. 27-28, 19 (2012).

[4] P. J. Dean, E. C. Lightowlers, and D. R. Wight, Phys. Rev. 140, A352 (1965).

[5] D. Labrie and T. Timusk, Solid State Commun. 53, 327 (1985).

[6] P. L. Gourley and J. P. Wolfe, Phys. Rev. B 25, 6338 (1982).

[7] T. Dekorsy, J. M. Sun, W. Skorupa, B. Schmidt, and M. Helm, Appl. Phys. A 78, 471 (2004).

[8] R. Corkish, D. S.-P. Chan, and M. A. Green, J. Appl. Phys. 79, 195 (1996).

[9] M. Nagai, R. Shimano, K. Horiuchi, and M. Kuwata-Gonokami, Phys. Rev. B 68, 081202(R) (2003).

[10] J. Omachi, T. Suzuki, K. Kato, N. Naka, K. Yoshioka, and M. Kuwata-Gonokami, Phys. Rev. Lett. 111, 026402 (2013).

[11] J. Barjon, E. Chikoidze, F. Jomard, Y. Dumont, M.-A. PinaultThaury, R. Issaoui, O. Brinza, J. Achard, and F. Silva, Phys. Status Solidi A 209, 1750 (2012).

[12] M. A. Pinault-Thaury, B. Berini, I. Stenger, E. Chikoidze, A. Lusson, F. Jomard, J. Chevallier, and J. Barjon, Appl. Phys. Lett. 100, 192109 (2012).

[13] M. A. Pinault-Thaury, I. Stenger, F. Jomard, J. Chevallier, J. Barjon, A. Traore, D. Eon, and J. Pernot, Phys. Status Solidi A 212, 2454 (2015).

[14] J. Barjon, F. Jomard, and S. Morata, Phys. Rev. B 89, 045201(2014).

[15] N. Naka, J. Omachi, H. Sumiya, K. Tamasaku, T. Ishikawa, and M. Kuwata-Gonokami, Phys. Rev. B 80, 035201(2009).
[16] P. Lefebvre, C. Brimont, P. Valvin, H. Miyake, K. Hiramatsu, and B. Gil, Phys. Status Solidi A 211, 765 (2014).

[17] J.R. Haynes, Phys. Rev. Lett. 4, 361 (1960).

[18] The initial exciton density is estimated to be about $2 \times$ $10^{14} \mathrm{~cm}^{-3}$, given an energy density per pulse of $0.1 \mu \mathrm{J} / \mathrm{cm}^{2}$ and assuming a $5 \times 10^{3} \mathrm{~cm}^{-1}$ absorption coefficient at $200 \mathrm{~nm}$ [S. Adachi, Optical Constants of Crystalline and Amorphous Semiconductors (Kluwer Academic, Boston, 1999)].

[19] W. Klingenstein and W. Schmid, Phys. Rev. B. 20, 3285 (1979).

[20] M. Kozák, F. Trojánek, and P. Malý, Phys. Status Solidi A 210 , 2008 (2013).

[21] H. Schlangenotto, H. Maeder, and W. Gerlach, Phys. Status Solidi A 21, 357 (1974).

[22] N. Naka, K. Fukai, Y. Handa, and I. Akimoto, Phys. Rev. B 88, 035205 (2013).

[23] H. Nakayama, T. Nishino, and Y. Hamakawa, Jpn. J. Appl. Phys. 19, 501(1980).

[24] M. Nakamura, T. Suzuki, and M. Fujita, Phys. Rev. B 35, 2854 (1987).

[25] R. Heitz, A. Hoffmann, and I. Broser, Phys. Rev. B 49, 14307 (1994).

[26] $\tau_{\mathrm{B}}$ can also be deduced from the delay between the maxima of free- and bound-exciton recombination intensities. Such an analysis provides comparable results given the measurement uncertainties.

[27] J. P. Bergman, C. I. Harris, O. Kordina, A. Henry, and E. Janzen, Phys. Rev. B 50, 8305 (1994).

[28] D. F. Nelson, J. D. Cuthbert, P. J. Dean, and D. G. Thomas, Phys. Rev. Lett. 17, 1262 (1966).

[29] W. Schmid, Phys. Status Solidi B 84, 529 (1977). 
[30] R. Sauer, N. Teofilov, K. Thonke, and S. Koizumi, Phys. Status Solidi A 201, 2405 (2004), and references therein.

[31] S. Rein, Lifetime Spectroscopy: A Method of Defect Characterization in Silicon for Photovoltaic Applications (Springer, Berlin, 2005).

[32] J. Barjon, T. Tillocher, N. Habka, O. Brinza, J. Achard, R. Issaoui, F. Silva, C. Mer, and P. Bergonzo, Phys. Rev. B 83, 073201 (2011).
[33] J. Barjon, P. Desfonds, M.-A. Pinault, T. Kociniewski, F. Jomard, and J. Chevallier, J. Appl. Phys. 101, 113701 (2007).

[34] I. Broussell, J. A. H. Stotz, and M. L. W. Thewalt, J. Appl. Phys. 92, 5913 (2002).

[35] J. Barjon, M.-A. Pinault, T. Kociniewski, F. Jomard, and J. Chevallier, Phys. Status Solidi A 204, 2965 (2007). 